\title{
The Mechanical Design and Fabrication of a Pneumatic Operated Deep Root Feeder and Subsurface Water Injector towards Efficient Irrigation
}

\author{
Robin David, Avinash G. S., Amal P. V., Anandhu J. S., Nithil Prasobh, Vishnu Sankar S. J.
}

\begin{abstract}
Agriculture is the significant wellspring of nourishment for mankind, for which irrigation system assumes an indispensable job. A portion of the primary sorts of irrigation techniques are surface irrigation, sprinkler irrigation and drip irrigation. It is the frequent application of small quantity of water directly, above or beneath the soil surface; ordinarily as discrete, persistent drops. Be that as it may, these current systems are pervious to numerous disadvantages and the major ones being evaporative loss, leaching loss and insufficient irrigation. In the vast majority of the present strategies, water doesn't reach down to the root zone which influences the efficiency and development of the yields. Micro irrigation additionally causes issues as the supply is given through pipes and ducts which when disintegrated could harm the soil.
\end{abstract}

In this paper a new system is designed with the intention of injecting water to the root zone. The proposed design involves a pneumatic cylinder onto which a hollow rod and nozzle arrangement is associated, a direction control valve, compressor, pump, solenoid flow controlling valve and a flow meter. Compressed air from compressor is utilized to control the pneumatic cylinder, and utilizing this power the hollow rod is penetrated into the soil. Water is conveyed at high velocity through the nozzle to the soil. The flow meter estimates the measure of water injected by revolution of a turbine. The sensor and microprocessor shows the amount digitally on LCD unit. The flow of water to the nozzle from the pump is constrained by means of a solenoid flow control valve. As the quantified measure of water is injected, the valve is actuated by the microprocessor to cut the water supply. The fabrication of the system is done as per the design and a user interface software is designed. The final design has been tested to check the efficiency of the system and from result it was clear that this framework helps in conquering the hindrances winning in the present procedures.

Index Terms - Irrigation, Micro irrigation, Irrigation efficiency, Drip irrigation, Pneumatic cylinder, Nozzle, Flow meter, Solenoid flow control valve

Manuscript revised June 10, 2019 and published on July 10, 2019

Robin David, Assistant Professor, Department of Mechanical Engineering, VAST TC, Kilimanoor, Affiliated to APJ Abdul Kalam Technological University, , Kerala, India

Avinash G. S Assistant Professor, Department of Mechanical Engineering, VAST TC, Kilimanoor, Affiliated to APJ Abdul Kalam Technological University, Kerala, India

Amal P. V., Anandhu J. S., Nithil Prasobh, Vishnu Sankar S. J., U. G Students, Department of Mechanical Engineering, VAST TC, Kilimanoor, Affiliated to APJ Abdul Kalam Technological University, , Kerala, India

\section{INTRODUCTION}

Irrigation is the process in which water is supplied to the plants by any artificial means like pipes, canals, sprinklers and so on. Agriculture cannot sustain without proper irrigation in places where the rainfall is scattered or seasonal. It has to be frequently done since the rainfall is not sufficient for the growth of crops. Irrigation has been practiced since ancient times and is still developing.

Restricted chances to grow the volume of worldwide freshwaters designated to water system implies that best in class water system innovations, expecting to improve productivity of existing frameworks are required, convenient, and are of fundamental importance. There is little extension for more noteworthy utilization of assigned worldwide freshwaters for water system, because of remarkable development since the 1950s, in addition to other different requests on that asset to satisfy higher living guidelines.

Globally, agribusiness is the biggest client of freshwater, with water system withdrawals speaking to roughly seventy-five percent of the absolute freshwater use. Enlargement techniques, for example, downpour water gathering, are important to support crisp water supplies. However, improving the productivity of utilization is of vital significance to help economical utilization of this asset. Agribusiness sits at the interface between the earth and society, so any expansion in water use should consider the subsequent effects on new water biological communities and the multifunctional nature and decent variety of advantages that flooded horticulture gives, not simply to nourishment generation. The benefit of utilizing new waters for water system ought to incorporate not just direct advantages to the gathering that stands to pick up from the item yet additionally the more extensive environmental outcomes of these choices, and the social objectives being served by the choice.

In accordance with the needs a literature survey was conducted and found many such projects but all those had many disadvantages and demerits. So we designed a new system in which many features were added and has the following: a system which could inject water along with fertilizer into the root zone, a flow meter which indicates the amount of water supplying to the root zone and thereby reduce the risk of over irrigation, a pneumatic piston and cylinder for easy penetration into the root zone

This paper mainly deals with the design of various components used in this system, calculation of force required for penetration into soil, programming of Arduino board for the controlling of solenoid valve, comparison with the 
existing techniques, fabrication of root feeder and sub soil water injector.

B. Niemoeller et al [1] discussed in detail about liquid injection into the soil with the aid of a high-pressure jet. The potential and the possibilities of injection by a high-pressure jet are being examined in trials on a stationary test rig. In these trials, different soils were used under different conditions (soil moisture, and soil density), and the possibilities of injecting pure water in the form of a high-pressure water jet are discussed.

Leonor. et al [2] explained about the subsurface drip irrigation. SDI is "the application of water below the soil surface though emitters, with discharge rates generally in the same range as drip irrigation." An SDI unit is simply a drip irrigation network buried at a certain depth. The efficiency of subsurface drip irrigation SDI could be similar to drip irrigation but it uses less water

Du Chang-long et al [3] discussed about the cutting efficiency and percentage of lumps for the auger mining machine. Here they selected the auger which has good cutting efficiency and percentage of lumps, Production efficiency and advance speed of new mechanism increased, and the axial resistance is reduced in the same conditions as the existing operating mechanism It is used for coal collapsing cutting with picks. Simultaneously, the percentage of lumps of the new operating mechanism is increased, while wear on the picks is uniform.

Brian J Mailloux et al [4] described, that there are mainly two types of injection system, a large tank and dynamic mixing tank which deliver more than 800Lof ground water which aquifer over 12 hours without changing the ground water temperature. Multi-level samplers of two types are designed and installed. MLS performed well for the homogenous surficial aquifer but their installation procedure promoted vertical mixing which could obfuscate experimental data obtained from vertically stratified, heterogeneous aquifers.

Pham Van Quang. et al [5] investigated by a research which was conducted on 10 citrus plantations at Hau Giang province in the Mekong delta, Vietnam with the use of a portable electronic penetrometer to study the impact of soil compaction in a time of one year. For measuring soil moisture content soil samples were taken at each $10 \mathrm{~cm}$ depth and soil penetration resistance was measured at range of 0-80 $\mathrm{cm}$. The results are PR increased as a function of raised-bed's age and it could be reached to a high degree by soil moisture variability during the season. Due to the age of raised beds sensitivity of PR to the soil decreases, while moisture represented an increasing tendency with the age of raised-beds.

\section{PROBLEM IDENTIFICATION}

From the literature review, it is observed that there are numerous problems in the present irrigation techniques. After further investigation on current scenario in irrigation, the following conclusions were drawn.

a. Large amount of water is lost as evaporative loss and irrigation efficiency is low in the existing irrigation methods.

b. Micro irrigation methods have high initial costs. It also requires experienced labour for its operation.

c. The amount of water supplied is not measured and hence there is a high chance for over watering.

d. The life of pipes and tubes used for irrigation is shortened due to the exposure to sunlight. When these get eroded, it causes soil pollution.

e. The water used for irrigation might contain debris and it leads to clogging.

f. There are chances of leach loss and thereby reducing the effect of fertilizer.

g. During surface irrigation the possibility of soil erosion is high.

\section{METHODOLOGY}

Design of Pneumatic Operated Root Feeder and Sub-Soil Water Injector system requires knowledge of Solid Mechanics, fluid dynamics, soil dynamics, electronic circuits, basic machine design and Thermal Engineering principles. The purpose of this chapter is to describe the guidelines of designing and fabricating of pneumatic cylinder, sub soil injector, connecting tubes, water quantity measuring unit and sensor unit. In this chapter, functions of each component in Pneumatic Operated Root Feeder and Sub-Soil Water system, mathematics involved with pneumatic cylinder design, sub soil injector design, and the design of various components of the system have been discussed elaborately.

\section{A. Working of pneumatic operated root feeder and sub-soil water injector}

The main components of this system are pneumatic cylinder, piston rod, direction control valve, nozzle, pump, air compressor, turbine flow meter, solenoid flow control valve, Arduino board, connecting hoses and a frame with handle. The pneumatic cylinder is being powered by an air compressor. A five way three position DCV is used for controlling the motion of the cylinder by directing the flow of compressed air. The piston rod is connected to a hollow shaft with nozzle by means of a $\mathrm{T}$ joint. A hose from pump is also connected to $\mathrm{T}$ joint. A frame along with a handle supports the cylinder. A solenoid valve is placed in hose near to handle along with a turbine flow meter 


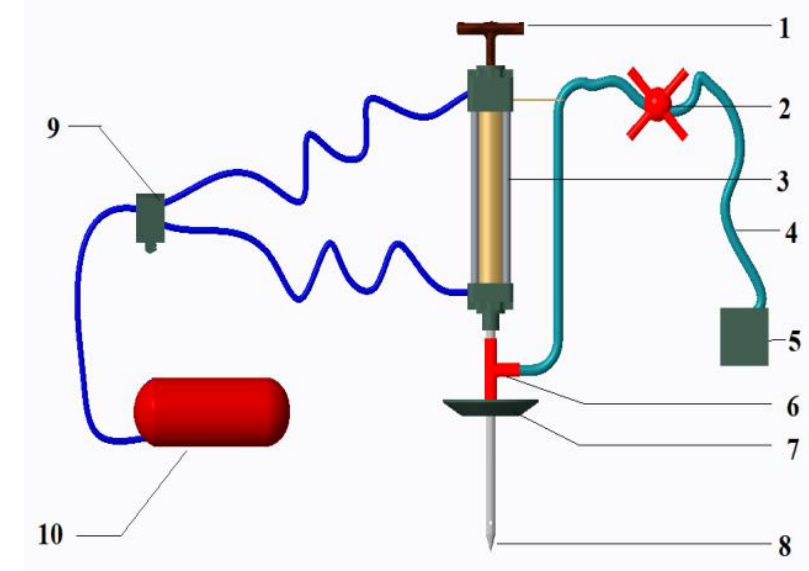

Fig.1: Block diagram of pneumatic operated root feeder and sub surface water injector

1. Handle

2. Flow regulating valve

3. Pneumatic cylinder

4. Connecting hose

5. Water pump

6. T joint

7. Foot rest stand

8. Nozzle

9. Direction control valve

10. Air compressor

The system is placed on the ground where the injection is to be done. The DCV is put to first position, and thus allowing compressed air to enter into the blank end of the cylinder head. The compressed air pushes the cylinder downward due to which the piston rod and hollow shaft moves downward. The handle is used to properly align and to balance the system. The force from the pneumatic system allows the hollow shaft to penetrate into the soil. As the rod reaches the required depth, the DCV is put to the second position, blocking the interaction between pneumatic cylinder and the compressor and the rod retains its position. Now the pump is switched on and the valve is opened, allowing a metered quantity of water to enter into the hollow shaft. The water flows through the nozzle, further increasing the velocity of water. This high energy water penetrates the soil and is accumulated near the root zone. Once the required value of water is injected, Arduino board detects the output from flow meter and actuates the solenoid valve to close and the supply of water is cut off. Now the DCV is put to the third position. In this position, compressed air enters into the rod end of the pneumatic cylinder and the cylinder along with the piston and hollow rod retracts.

Main components used for Pneumatic Operated Root Feeder and Sub-Surface Water Injector are:
a. Pneumatic cylinder
b. Directional Control Valve
c. Nozzle

\author{
d. Air Compressor \\ e. Flow meter \\ f. Water Pump \\ g. Flow regulating valve \\ h. Arduino board \\ i. Connecting hose
}

\section{B. Theoretical aspects of components used}

a. Pneumatic cylinders: These are mechanical devices which utilize the power of compressed gas to deliver a force in a reciprocating linear movement. The function of pneumatic cylinder in this project is to power the system. It provides the linear motion of rod with sufficient force required for penetration.

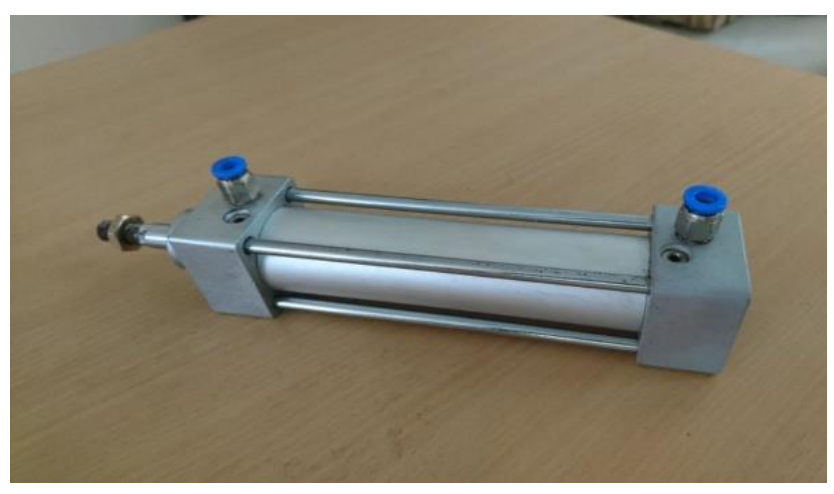

Fig.2: Pneumatic cylinder

a. b. Direction Control Valve: The function of directional control valve is to control the heading of flow in the pneumatic circuit. DCVs are utilized to begin, stop and control the heading of air flow and to help in the distribution of air in the required line. It is DCV which helps in the control of motion of the rod. In this paper a 5 way 3 position $(5 / 3)$ DCV is used.

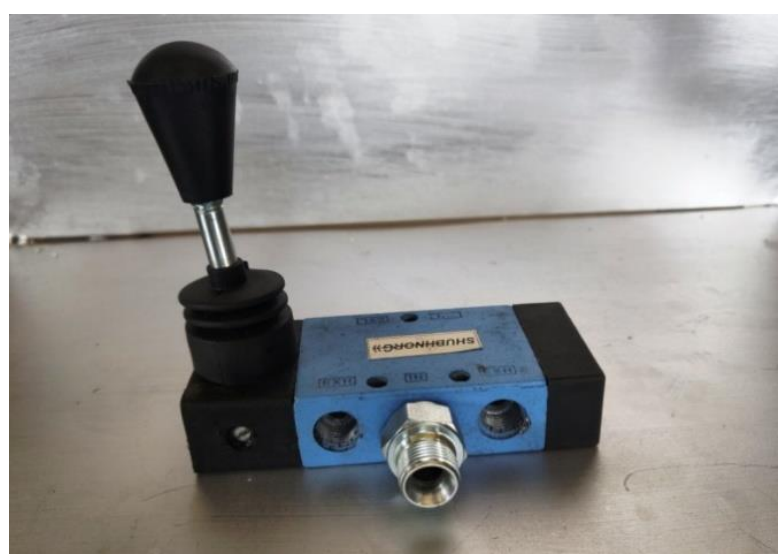

Fig. 3: Direction Control Valve

c. c. Nozzles: A nozzle is regularly a pipe or container of differing cross sectional region and it very well may be utilized to coordinate or change the flow of a liquid such as rate of flow, speed, direction, mass, shape, as well as the pressure of the stream that emerges from them. A nozzle with minute holes on periphery which allows $360^{\circ}$ supply of water at high velocity is used in this paper. 


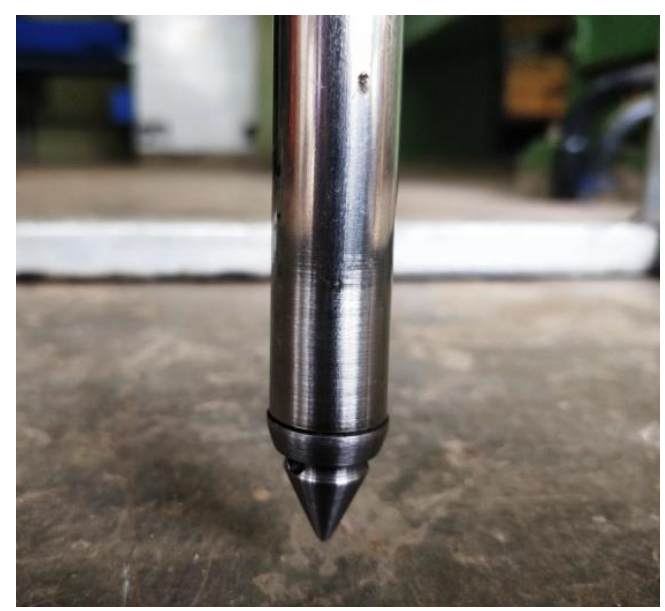

Fig. 4: Nozzle

d. Air Compressor: An air compressor is a device that converts power into potential energy put away in pressurized air. A mobile compressor unit having a capacity of 9 bar is used in this paper. The purpose of compressor is to deliver compressed air for the working of pneumatic cylinder.

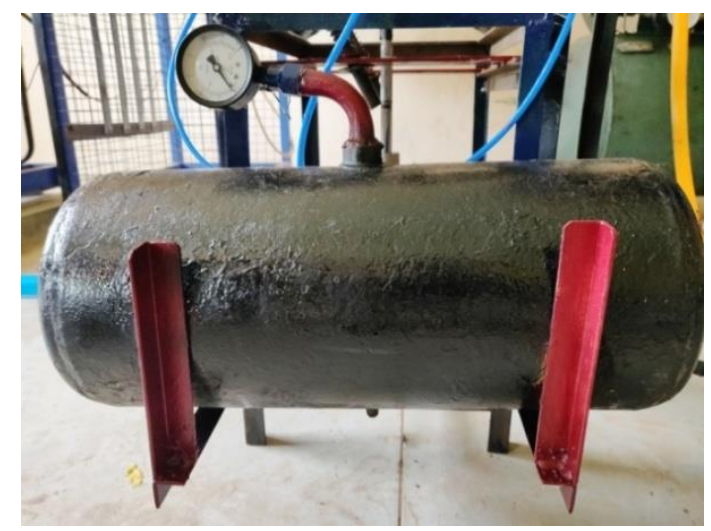

Fig. 5: Air compressor

e. Flow meter: Flow meters are gadgets that measure the amount of fluid, gas or vapour that goes through them. Turbine flow meter having digital display is used in this paper. The amount of water injected by the system can be monitored by flow meter. The output from flow meter is in turn used in Arduino to actuate the solenoid valve.

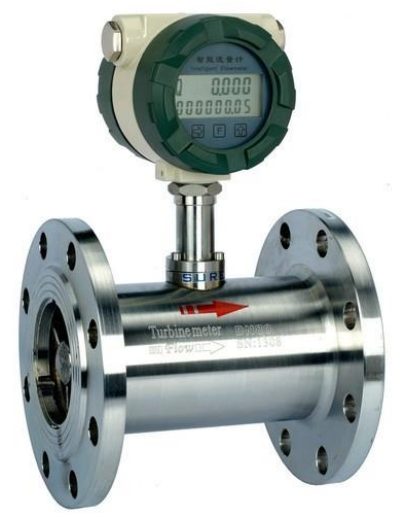

Fig. 6: Turbine type Flow meter f. Water Pump: A pump is a device that moves liquids or some of the time slurries, by mechanical activity. In this work a centrifugal pump is used in which pumping action is due to forced vortex

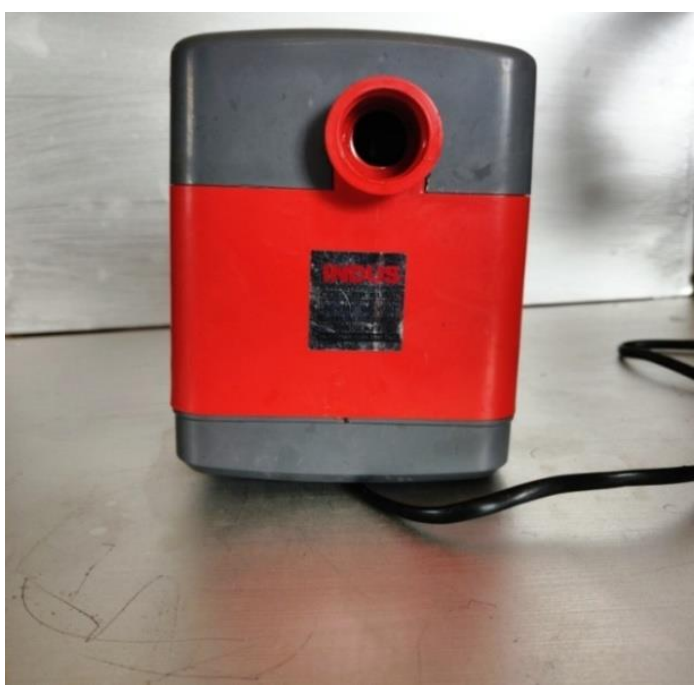

Fig. 7: Centrifugal Water Pump

g. Flow Regulating Valve: A flow control valve directs the flow or pressure of a liquid. Control valves typically react to signals produced by independent gadgets, for example, flow meters or temperature checks. Solenoid flow control valve is utilized in this paper. The actuation of solenoid valve is being carried out by Arduino board.

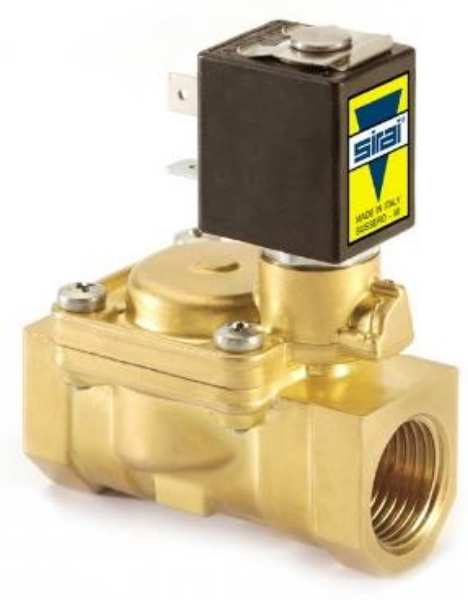

Fig. 8: Solenoid Flow Control Valve

h. Arduino Board: The Arduino Uno is an open-source microcontroller board based on the Microchip ATmega328P microcontroller and developed by Arduino.cc. The board is equipped with sets of digital and analog input/output (I/O) pins that may be interfaced to various expansion boards and other circuits. In this paper we make use of Arduino Uno for controlling the solenoid flow control valve. 


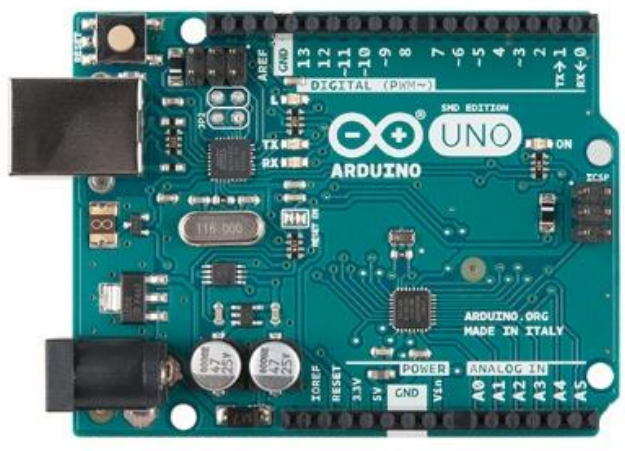

Fig. 9: Arduino board

i. Connecting Hoses: Hoses used to convey consumable water are normally made of NSF International-recorded polymers. Hoses are being used to convey water and compressed air in this work.

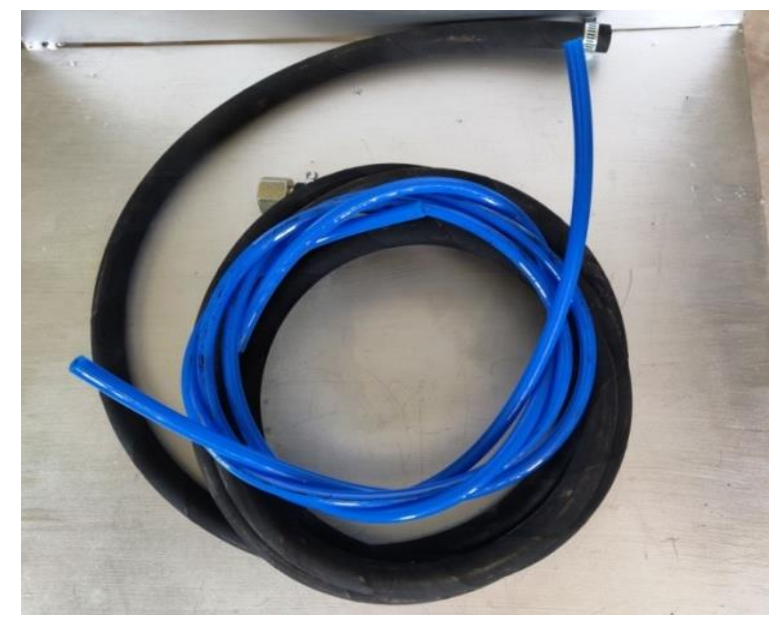

Fig. 10: Connecting Hoses

\section{DESIGN}

For the design process the soil dynamics is considered. From the load bearing, test penetration resistance and shear stress of the sample soil is calculated. The diameter of the rod is assumed to be in the range of $2-5 \mathrm{~cm}$ in diameter. Using the above data, the force and pressure required for the penetration of the rod is calculated, also a factor of safety of 6 is considered for the design process. The value of pressure obtained is standardized in order to design the pneumatic cylinder. The connecting hoses and direction control valve is also selected based on the pneumatic cylinder. Programming is done on Arduino board using $\mathrm{ClC}++$ language. A user interface will be provided such that the amount of water to be injected can be set by the user according to the crop.

As a part of this work, we have designed the following:

a) Pneumatic cylinder

- Maximum pressure requirement

- Stroke length

- Bore
b) Hollow rod
- Diameter
- Thickness
- Length
c) Nozzle
d) Frame
e) $\mathrm{T}$ junction

This project involves penetration of a rod into soil by means of pneumatic cylinder. For this, penetration resistance of the soil is to be calculated and for the calculation purpose values from the static cone penetration test in Soil Mechanics and the Foundation Engineering (by A Arora) is taken.

Assume the penetration depth to be $300 \mathrm{~mm}$. By some research we have arrived at the conclusion that, for up to $1 \mathrm{~m}$ depth, profile of soil will be silty soil which has medium consistency. Thus we have to find penetration resistance by considering profile of soil as silts and clayey silts type with medium consistency and loose dense soil.

For this particular type soil;

Resistance experienced at the cone tip.

Where,

$$
\mathrm{q}_{\mathrm{c}} \quad=(200-400) \times \mathrm{N}
$$

$\mathrm{N}$ - Standard penetration number

For soil with medium consistency, the Values of $\mathrm{N}$ lies between $4-5$

Assume;

$$
\begin{aligned}
& \mathrm{q}_{\mathrm{c}}=200 \mathrm{~N} \mathrm{kN} / \mathrm{m}^{2} \\
& \mathrm{~N}=4
\end{aligned}
$$

Therefore, $\mathrm{q}_{\mathrm{c}}=800 \mathrm{kN} / \mathrm{m}^{2}$

$$
=8 \mathrm{bar}
$$

By standardizing pneumatic cylinder having capacity of 10 bar pressure and $50 \mathrm{~mm}$ bore is selected.

Force exerted on hollow rod, $\mathrm{F}=P \times A$

$\mathrm{P}=$ Pressure acting on cylinder $=8 \mathrm{bar}=0.8 \mathrm{~N} / \mathrm{m}^{2}$

$\mathrm{A}=$ Area of cylinder $=\frac{\pi}{4} \times 50^{2}=1963.5 \mathrm{~mm}^{2}$

$$
F=0.8 \times 1963 .=1570.8 \mathrm{~N}
$$

Working stress, $\sigma_{\mathrm{w}}=F / a$

Where,

$$
\begin{aligned}
& \mathrm{a}=\text { Cross sectional area of } \operatorname{rod}=\frac{\pi}{4} \times d^{2} \\
& \mathrm{~d}=\text { Diameter of rod }
\end{aligned}
$$

Assume diameter of rod to be $25.4 \mathrm{~mm}$ and thickness 2.108 $\mathrm{mm}$

$$
\begin{array}{ll}
\mathrm{a} & =\frac{\pi}{4} \times\left(25.4^{2}-21.84^{2}\right)=132.08 \mathrm{~mm}^{2} \\
\sigma_{\mathrm{w}} & =\frac{1570.8}{132.08}=11.89 \mathrm{~N} / \mathrm{mm} 2
\end{array}
$$

Here we use stainless steel rod having yield stress of 270 $\mathrm{MPa}$, and a factor of safety of 6 is taken.

$$
\sigma_{\mathrm{m}}=\frac{\text { Yieldstress }}{\text { Factorofsafety }}=\frac{270}{6}=45 \mathrm{~N} / \mathrm{mm}^{2}
$$


It was observed that the working stress of material is within the maximum allowable stress. Thus the design is safe.

\section{A. Part Drawing using PTC CREO}

The components required for the project is modeled in PTC Creo. The 3D models of the components are as follows.

\section{a. Pneumatic Cylinder}

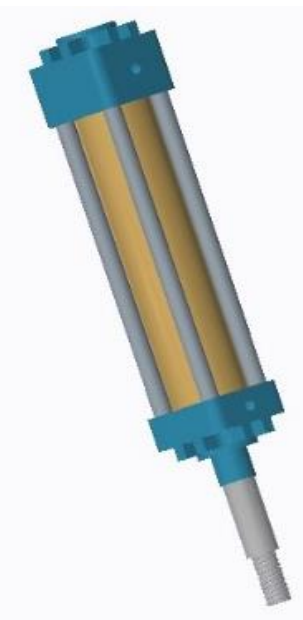

Fig. 11: Pneumatic cylinder

b. T joint

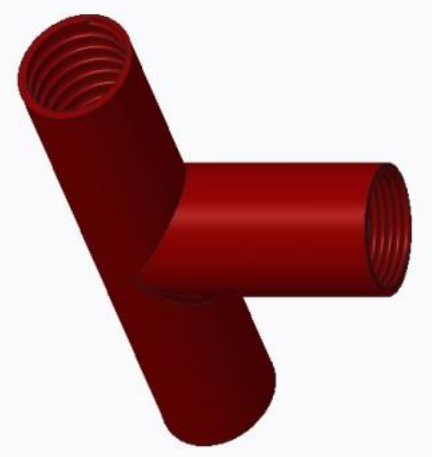

Fig. 12: T Joint

\section{c. Handle}

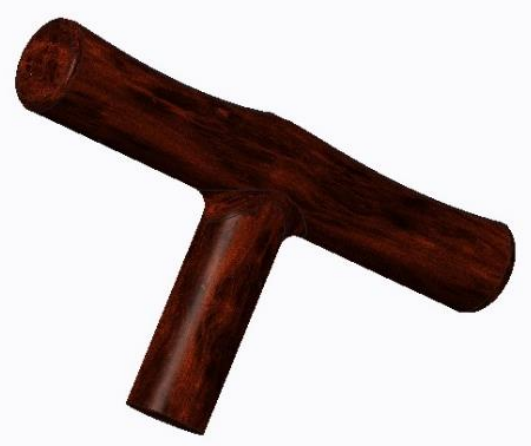

Fig. 13: Handle

\section{d. Hollow rod}

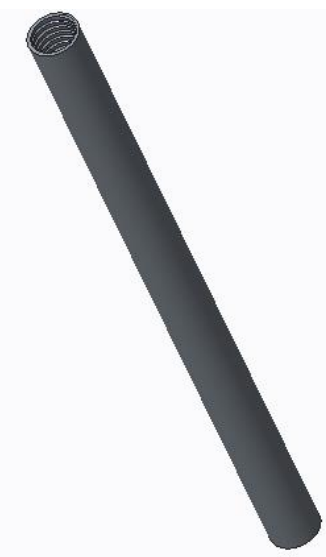

Fig. 14: Hollow rod

\section{RESULTS AND DISCUSSION}

The 3D model of the pneumatic root feeder has been designed using PTC Creo. The required standardized components for the project include pneumatic cylinder, direction control valve, stainless steel rod, pump, compressor, connecting hoses, nozzle, flow control valve, flow meter etc. Using these, the various parts of the system were designed. After designing the injector system parts, the detailed dimensioned drawing of the system was done. Then the fabrication of the whole project was done and it's testing was done on silty soil.

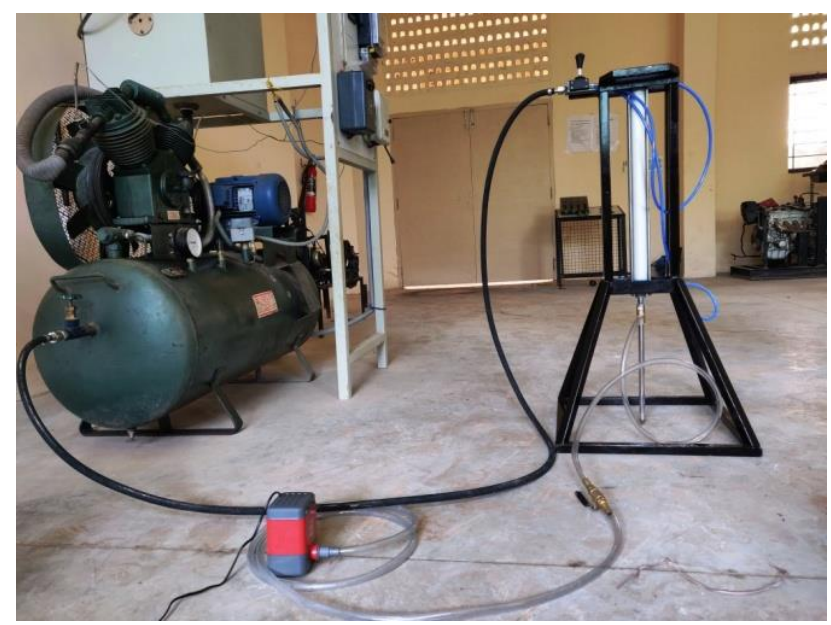

Fig. 15: Assembled view of Prototype

After fabricating the system, we tested it for evaluating the results. The test procedure and observations as follows;

$>$ The connection from compressor to direction control valve and from direction control valve to pneumatic cylinder is done by means of connecting hoses.

$>$ The system is placed in position where the injection is to be performed and injection is done by operating DCV and flow control valve.

$>$ After operating the system, water content of the soil is analysed. 
$>$ The water content of soil has been increased.

$>$ There was no sign of leach loss or evaporative loss.

$>$ By creating a disturbance in the soil, chance of capillary rise has been eliminated.

\section{CONCLUSIONS}

The project, pneumatic operated root feeder and subsoil water injection is an advanced type water irrigation system. The implementation of the system was very smooth, easy and very effective at a very low cost compared to all other water irrigation techniques. It could give an effective utilization of water. Liquid fertilizer also can be applied along with water. Comparatively the time taken for operation is very less. The quantity of water that depends on the crops can be controlled by flow regulating valve. It enhances the growth rate of plants contrast to other techniques. As the root feeder system directly injects water beneath the soil, the frequency of watering the plant is minimized and the frequency varies as per the requirement of the particular plant.

The test for the work has been conducted by operating the system by compressed air and performing the function on silty soil and thus found that enough quantity of water could be injected into the soil with an expenditure of adequate time. A system which digitally displays the amount of water injected has been designed, by which any chances of over irrigation or under irrigation is eliminated. We have arrived at the conclusion that this system is capable of saving water and fertilizer; it also greatly boosts the growth rate of plant. The chances of leach loss and evaporative loss have been eliminated completely along with increasing the irrigation efficiency to $100 \%$, as the complete water supplied has been injected beneath the soil. Thus, it is an efficient device made by keeping in mind about the difficulties and challenges in soil irrigation.

\section{REFERENCES}

[1] B. Niemoeller, H. H. Harms, T. Lang "Injection of liquids into the soil with a high-pressure jet”, June, 2011 Agric EngInt: CIGR Journal Vol. 13, No.2

[2] Leonor Rodriguez Sinobas and Maria Gil Rodriguez "A Review of Subsurface Drip Irrigation and Its Management", Chapter - March 2012DOI: $10.5772 / 30702$ - Source: In Tech

[3] Du Chang-long, Liu Song-yong, Cui Xing-Xia, Gu Jiu-lin" Study on design of operating mechanism of auger mining machine", Procedia Earth and Planetary Science 1 (2009) 1406-1410

[4] Brian J. Mailloux, Mark E. Fuller, George F. Rose, Tullis C. Onstott, Mary F. De Flaun, Enrique Alvarez, Chris Hemingway, R. Bruce Hallet, Tommy J. Phelps, and Timothy Griffin "A Modular Injection System, Multilevel Sampler, and Manifold for Tracer Tests" vol. 41, no. 6-ground water- November-December 2003

[5] Pham Van Quang, Per-Erik Jansson and Le Van Khoa "Soil Penetration Resistance and Its Dependence on Soil Moisture and Age of the Raised-Beds in the Mekong Delta, Vietnam", international journal of engineering research and Development ISSN: 2278-067X pISSN: 2278-800X, Volume 4, Issue 8 (November 2012), PP. 84-93

\section{AUTHORS PROFILE}

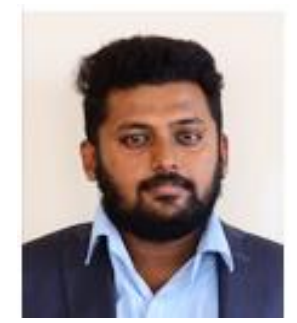

Mr. Amal P. V. pursuing B. Tech in Mechanical Engineering, $4^{\text {th }}$ year $8^{\text {th }}$ semester from Vidya Academy of Science and Technology, Technical Campus, Kilimanoor, Kerala, India

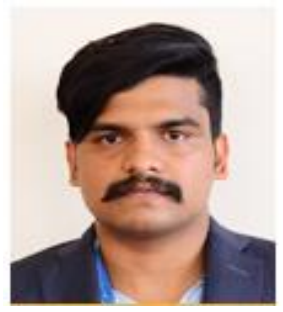

Mr. Anandhu J. S., pursuing B. Tech in Mechanical Engineering, $4^{\text {th }}$ year $8^{\text {th }}$ semester from Vidya Academy of Science and Technology, Technical Campus, Kilimanoor, Kerala, India
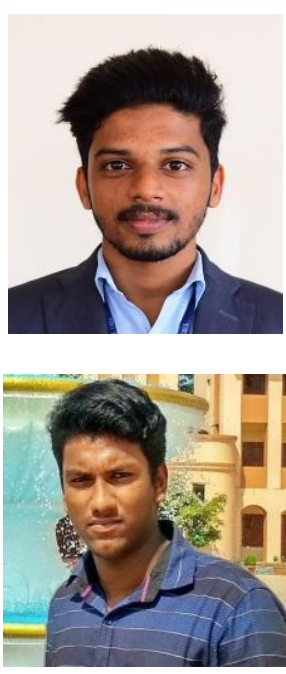

Mr. Nithil Prasobh., pursuing B. Tech in Mechanical Engineering, $4^{\text {th }}$ year $8^{\text {th }}$ semester from Vidya Academy of Science and Technology, Technical Campus, Kilimanoor, Kerala, India

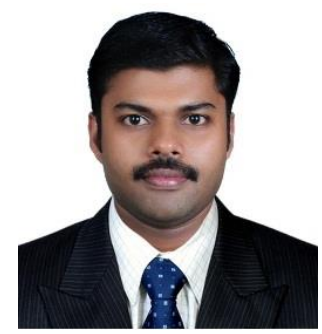

Mr. Vishnu Sankar S. J., pursuing B. Tech in Mechanical Engineering, $4^{\text {th }}$ year $8^{\text {th }}$ semester from Vidya Academy of Science and Technology, Technical Campus, Kilimanoor, Kerala, India

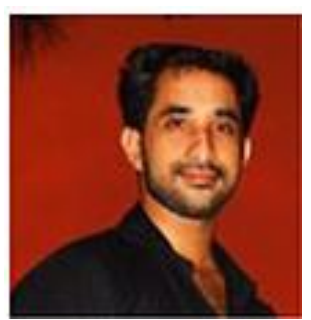

Mr. Robin David presently working as Asst. Professor in Vidya Academy of Science and Technology, Technical Campus, Kilimanoor, Kerala, India. He did his M. tech in Automobile Engineering from NIT Warangal and B. Tech from T.K.M College of Engineering, Kollam, Kerala. He is having a total teaching experience of around 6 years in various renowned institutions. 